\title{
Clinical study of controlling myopia progression of students wearing orthokeratology
}

\author{
Chunyan Zhang ${ }^{1,2}$, Lili Gao ${ }^{1}$, Xiangyang Xin*1 \\ ${ }^{1}$ Department of Ophthalmology, the Third Affiliated Hospital, Baotou, China \\ ${ }^{2}$ Inner Mongolia Medical University, Hohhot, China
}

Received: January 13, 2017

DOI: $10.14725 /$ dcc.v4n1p9

\author{
Accepted: February 18, $2017 \quad$ Online Published: March 10, 2017 \\ URL: http://dx.doi.org/10.14725/dcc.v4n1p9
}

\begin{abstract}
Objective: To observe the clinical effects of wearing orthokeratology lenses to control the myopia progression of students. Methods: 105 cases (10-16 years) from January 2011 to October 2014 with low, moderate myopia were selected, the spherical equivalent was (-3.63 \pm 0.95$)$ D. 56 cases (112 eyes) were wearing orthokeratology lenses, 49 cases (98 eyes) were wearing glasses. Both groups were followed up for 2 years to observe the variations of spherical equivalent, axial length, intraocular pressure and corneal thickness.

Results: The spherical equivalent changes after two years wearing: the spherical equivalent of the orthokeratology lenses group increased by $(-0.47 \pm 0.98) \mathrm{D}$, and that of the glasses group increased by $(-1.59 \pm 0.91) \mathrm{D}$; the difference was statistically significant $(p<.05)$. The axial length changes after two years wearing: the axial length of the orthokeratology lenses group increased by $(0.17 \pm 1.09) \mathrm{mm}$, and that of the glasses group increased by $(0.72 \pm 1.08) \mathrm{mm}$, the difference between two groups was statistically significant $(p<.05)$. The variations of intraocular pressure and corneal thickness were not statistically significant before and after wearing lenses in the orthokeratology lenses group $(p>.05)$.

Conclusions: Wearing orthokeratology lenses can effectively delay the progression of myopia and control axial growth. The lenses can be disseminated and used in clinical practice.
\end{abstract}

Key Words: Orthokeratology, Myopia progression, Axial length

Myopia is a kind of refractive errors with highest incidence. ${ }^{[1]}$ The past decade has witnessed the incidence of myopia in children and adolescents and an increasing proportion of high myopia with a trend of early onset and rapid progress. ${ }^{[2]}$ It has become a clinical consensus that corneal shaping can control the progress of myopia so that the exploration of its mechanism has become a hot spot of research. ${ }^{[3]}$ The role of orthokeratology in the control of myopia growth has been confirmed by more and more literatures. ${ }^{[4]}$ In the present study, 105 cases of myopia treated with orthokeratology and frame glasses in our clinic were followed up and observed for 2 years, the results are reported as below.

\section{Data and methods}

\subsection{Study object}

A total of 105 young patients with myopia admitted to our hospital from January 2011 to October 2014 were enrolled in this study. Their ages ranged from 10 to 16 years (13.36 \pm 1.22 years $)$. The equivalent spherical degree was $(-3.63$ $\pm 0.95) \mathrm{D}$, including 48 males (96 eyes) and 57 females (114 eyes). The best corrected visual acuity was $\geq 1.0$ in all patients. No strabismus and eye diseases were observed.

\footnotetext{
*Correspondence: Jinghui Zhang; E-mail: xinxiangyang_2006@163.com; Address: Department of Ophthalmology, the Third Affiliated Hospital, Baotou, China.
} 
Compliance was good. Among them, 56 cases were wearing orthokeratology lenses (112 eyes) and 49 cases wearing frame glasses (one-half lenses were reviewed after a half year follow-up).

\subsection{Methods}

All patients were tested with standard logarithmic visual acuity chart for naked eyesight and corrected visual acuity. Subjective optometry was performed by CV-300 Refractor. The best corrected visual acuity and binocular balance were achieved, and myopia diopter was finally determined. The axial length of the ocular axis was measured 4 times per eye with CineScan ophthalmology A/B ultrasound, and the average value was taken. The corneal thickness was measured by Pacline corneal thickness instrument 6 times per eye, and the average value was taken. Intraocular pressure was measured 3 times each eye by using a non-contact intraocular pressure measuring instrument and the average value was taken.

Orthokeratology patients followed with the fitting procedures, routine examination of anterior and posterior segments of the exclusion of contraindications. The parameters measured by the corneal topographic map were tested and evaluated, and the location and mobility of the center were observed. According to the fluorescein staining static evaluation, the final confirmation of the stator parameters was made. Use the US E\&E night wear orthokeratology lens. 1 weeks, 1 month, 3 months, 6 months after wearing orthokeratology, the reexamination was carried out every half a year. 4 weeks discontinued after wearing lenses for 2 years, the corneal thickness, ocular axis and intraocular pressure were measured.

\subsection{Statistical analysis}

All the data in this study were recorded in the computer and processed using SPSS 13.0 statistical software. Independent sample $t$-test was used for comparison of independent parameters between the two groups, the same parameters before and after wearing in the two groups were compared using paired $t$-test for statistical analysis. The difference of $p<.05$ was statistically significant.

\section{Results}

\subsection{Basic information}

The difference of patients' age, spherical equivalent, and axial length of the two groups was not statistically significant $(p>.05)$ (see Table 1).

\subsection{Orthokeratology lenses group}

53 patients were wearing orthokeratology lenses for the whole course in 56 cases, accounting for $94.64 \%$. The equivalent spherical degree was $(-3.52 \pm 1.23) \mathrm{D}$ before wearing lenses, and $(-4.03 \pm 1.26) \mathrm{D}$ four weeks discontinued after wearing lenses for 2 years. The axial difference was statistically significant $(p<.05)$. The axial length of the ocular axis was $(24.62 \pm 1.22) \mathrm{mm}$ and $(24.81 \pm 1.36) \mathrm{mm}$ respectively before and after wearing lenses, the difference was statistically significant $(p<.05)$; the corneal thickness was $(558.59 \pm 23.71) \mu \mathrm{m}$ and $(553.46 \pm 25.67) \mu \mathrm{m}$ respectively before and after wearing lenses, the difference was not statistically significant $(p>.05)$. There was no significant difference in intraocular pressure before and after wearing lenses $(p>.05)$.

Table 1: Comparison of patients age, spherical equivalent, and axial length between two groups $(\bar{x} \pm \mathrm{s}, \mathrm{n}=105)$

\begin{tabular}{llll}
\hline Group & Age (year) & Equivalent spherical degree (D) & Axis length (mm) \\
\hline Orthokeratology lenses group & $13.50 \pm 1.70$ & $-3.52 \pm 1.23$ & $24.62 \pm 1.22$ \\
Glasses group & $13.30 \pm 1.20$ & $-3.65 \pm 0.98$ & $24.67 \pm 1.18$ \\
$t$ & 6.54 & 5.29 & 7.16 \\
$p$ & $>.05$ & $>.05$ & $>.05$ \\
\hline
\end{tabular}

Table 2: Changes of the equivalent sphere, axial length, corneal thickness and intraocular pressure before and after wearing lenses $(\bar{x} \pm \mathrm{s})$

\begin{tabular}{lllll}
\hline Time & Equivalent spherical degree $(\mathbf{D})$ & Axis length $(\mathbf{m m})$ & Corneal thickness $(\boldsymbol{\mu m})$ & Intraocular pressure $(\mathbf{m m H g})$ \\
\hline Before & $-3.52 \pm 1.23$ & $24.62 \pm 1.22$ & $558.59 \pm 23.71$ & $14.56 \pm 2.47$ \\
After & $-4.03 \pm 1.26$ & $24.81 \pm 1.36$ & $553.96 \pm 25.67$ & $14.39 \pm 2.34$ \\
$t$ & -9.03 & -5.11 & 5.48 & 8.31 \\
$p$ & $<.05$ & $<.05$ & $>.05$ & $>.05$ \\
\hline
\end{tabular}


Table 3: Changes of equivalent spherical degree and axial length before and after wearing frame glasses $(\bar{x} \pm \mathrm{s})$

\begin{tabular}{lll}
\hline Time & Equivalent spherical degree $(\mathbf{D})$ & Axis length $(\mathbf{m m})$ \\
\hline Before & $-3.65 \pm 0.98$ & $24.67 \pm 1.18$ \\
After & $-5.31 \pm 1.26$ & $25.40 \pm 1.26$ \\
$t$ & -10.62 & -9.61 \\
$p$ & $<.05$ & $<.05$ \\
\hline
\end{tabular}

\subsection{Frame glasses group}

The equivalent spherical degree before wearing glasses was $(-3.65 \pm 0.98) \mathrm{D}$, and $(-5.31 \pm 1.12) \mathrm{D}$ after two years, the difference was statistically significant $(t=-10.62, p<.05)$. The axial length of the ocular axis was $(24.67 \pm 1.18) \mathrm{mm}$ and $(25.40 \pm 1.26) \mathrm{mm}$ before and after the wearing glasses. The difference was statistically significant $(t=-9.61, p<$ .05) (see Table 3).

\subsection{Comparison of myopia diopter and axial length of the orthokeratology lenses group and the glasses group}

The myopic dioptre of the orthokeratology lenses group increased by $(-0.47 \pm 0.98) \mathrm{D}$ after 2 years, and that of the glasses group increased by $(-1.59 \pm 0.91) \mathrm{D}$, the difference was statistically significant $(t=29.66, p<.05)$. The axial length of the orthokeratology group increased by $(0.17 \pm$ $1.09) \mathrm{mm}$, and that of the glasses group increased by $(0.72$ $\pm 1.08) \mathrm{mm}$, the difference was statistically significant $(t=$ $27.43, p<.05)$ (see Table 4).

Table 4: Comparison of myopia diopter and axial changes $(\bar{x} \pm \mathrm{s})$

\begin{tabular}{lll}
\hline Group & Equivalent spherical degree(D) & Axis length $(\mathbf{m m})$ \\
\hline Orthokeratology lenses group & $-0.47 \pm 0.98$ & $0.17 \pm 1.09$ \\
Glasses group & $-1.59 \pm 0.91$ & $0.72 \pm 1.08$ \\
$t$ & 29.66 & 27.43 \\
$p$ & $<.05$ & $<.05$ \\
\hline
\end{tabular}

\section{Discussion}

It is a hot topic in the medical field to control the progress of students' myopia. Different approaches have been attempted clinically, including hard corneal contact lenses, orthokeratology lenses and multi-focus frame glasses. Orthokeratology is a kind lens whose base curve is 4-5 D flat than corneal central curvature. It produces a certain pressure on the cornea surface. According to the constant volume principle and the curvature of the reverse arc, it produces negative pressure pull in the space formed outside the optical area, making the center of the cornea flattened, so as to reduce the effect of myopia. ${ }^{[5]}$

The clinical efficacy in the control of myopia has been recognized. In recent years, there are many reports about the use of orthokeratology in controlling myopia. Cho et al. ${ }^{[4]}$ firstly found that after 2 years of wearing orthokeratology, the axial growth of the orthokeratology and frame glasses were $0.29 \mathrm{~mm}$ and $0.54 \mathrm{~mm}$. The results of this study were $0.17 \mathrm{~mm}$ and $0.72 \mathrm{~mm}$, the difference was statistically significant $(p<.05)$. And KaKita et al. ${ }^{[6]}$ reported that $0.22 \mathrm{~mm}$ axial phase continued to increase after wearing orthokeratology lenses for 2 years. Walline et al. ${ }^{[7]}$ showed that the axial length of children wearing orthokeratology for myopia increased by $0.32 \mathrm{~mm}$ in 2 years. Meanwhile, it was considered that the relative central fovea of peripheral retina was myopic defocus, which might prevent eye axis growth. A foreign study pointed out that 5-year axial growth of wearing orthokeratology reduced by $31 \%$ than that of glasses. ${ }^{[8]}$ Thus, the function of wearing orthokeratology lenses to slow down axial growth was confirmed by clinical research. After 2 years of study, Santodomingo-Rubido et al. ${ }^{[9]}$ also found that the axial growth of the orthokeratology and the glasses group were $0.47 \mathrm{~mm}$ and $0.69 \mathrm{~mm}$ respectively. There are also studies showing that: ${ }^{[3]}$ even with different races and different lens designs, the positive effect of orthokeratology on children's myopia intervention and the mitigation effect of axial growth could be replicated.

The basic theory of orthokeratology lens not only focuses on alleviating central retinal defocus, but also myopic defocus changes in the peripheral retina. ${ }^{[10]}$ Scholars ${ }^{[11]}$ believed that orthokeratology lenses may play a role in controlling the progression of myopia by producing peripheral myopic defocus. Whether it is the progression of myopia caused by peripheral refractive hyperopia and defocus, or the change of the shape of eyeball caused by myopia progression, the order of peripheral refraction is still controversial. ${ }^{[12]}$ But the number of further studies to investigate the different methods to change the peripheral refractive status and then to intervene in the progress of myopia is still increasing. Stone et al. ${ }^{[13]}$ suggested that for the peripheral retina, the partial axis imaging was relatively hyperopic. Even if the central focus was used, the local eyeball would grow properly, so that the peripheral retina could focus imaging. Conversely, due to biomechanics and the overall shape of the 
eyeball, the fovea often maintains or develops hyperopia if the off-axis refractive state is myopia.

Orthokeratology changes the center of the cornea and the refraction of the paracentesis, leaving the surrounding retina myopic defocused while maintaining focus in the central area. ${ }^{[14]}$ In Niu Y et al's study, ${ }^{[15]}$ the increase of the equivalent lenses after 1 year in the orthokeratology lenses group and the glasses group was $(0.31 \pm 0.21) \mathrm{D}$ and $(0.77 \pm$ $0.46) \mathrm{D}$ respectively. The results of our study showed that the equivalent lenses of the orthokeratology group and the glasses group increased by $(-0.47 \pm 0.98) \mathrm{D}$ and $(-1.59$ $\pm 0.91) \mathrm{D}$ respectively in 2 years. Orthokeratology lens has advantages over frame glasses in regard to high imaging quality, less hysteresis of the eye, and improved ocular conditioning, which may be the factors that slow down the progress of myopia. The visualization of the eye depends on the retinal imaging quality and optical defocus affects the axial length of the eye, leading to the growth of the eye and the development of myopia.

Orthokeratology lens has less accommodative hysteresis than glasses under different accommodative stimuli, especially in the case of high accommodative demands. ${ }^{[16]}$ The difference in adjusting hysteresis may be the result of a different diopter progression. Wu XY et al. ${ }^{[17]}$ showed that the defect of correction of myopia by frame glasses was over- come after wearing orthokeratology lenses, such as eliminating narrow frame glasses on the image. Secondly, the prism effect and the lens effect of the frame glasses are eliminated, and the demand for adjustment is increased. The increasing regulation demand causes the adjustment not be lasting, which leads to the lag of regulation, and the adjustment lag can induce the development of myopia. This may be the advantage of the orthokeratology lens in correcting students' myopia.

As for the safety of orthokeratology, occasional cornea with mild exfoliation was found in a few cases after a 2-year follow-up. It still obtains higher satisfaction when stop wearing one night with appropriate use of growth factors. Long-term studies have shown that modern orthokeratology lenses with high oxygen permeability also impose strong safety. ${ }^{[18]}$ In summary, the orthokeratology lens has a significant effect on reducing myopia and alleviating the progress of myopia. It is also reversible. It is indeed an effective way to control the progress of myopia as long as compliance with the fitting procedure, appropriate indications, follow-up reviews and an in-time handle of the problem.

\section{Conflicts of Interest Disclosure}

The authors have no conflicts of interest related to this article.

\section{References}

[1] Zhao J, Mao J, Luo R, et al. The progression of retractive error in school age children: Shunyi District, China. Am J Ophthalmol. 2002; 134(5): 735-743. https://doi.org/10.1016/ S0002-9394 (02) 01689-6

[2] Jiang J, Chen YY, Wu Ge, et al. Effect of different correction methods on the control of myopia in children. Chinese Journal of Optometry Ophthalmology and Visual Science. 2014; 16(2): 73-77.

[3] Zhu MJ, Feng HY, Zhu JF, et al. Effect of adjustment amplitude on myopia control in keratoplasty. Chinese Journal of Ophthalmology. 2014; 50(1): 14-19. PMid: 24709128.

[4] Cho P, Cheung SW, Edwards M. The longitudinal orthokeratology research in children (LORIC) in Hong Kong: a pilot study on refractive changes and myopia control. Curr Eye Res. 2005; 30(1): 71-80. PMid: 15875367. https://doi.org/10.1080/ 02713680590907256

[5] Qu XM, Chu RY. Clinical effect and related factors of keratology. Chinese Journal of Optometry \& Ophthalmology. 2004; 6(1): 6-9.

[6] KaKtia T, Hiraoha T, Oshika T, et al. Influence of overnight orthokeratology on axial length elongation in childhood myopia. Invest Ophthalmol Vis Sci. 2011; 52(5): 2170-2174. PMid: 21212181. https://doi.org/10.1167/iovs.10-5485

[7] Walline JJ, Jones LA, Sinnott LT, et al. Corneal reshaping and myopia progression. Br J Ophthalmol. 2009; 93(9): 1181-1185. PMid: 19416935. https://doi.org/10.1136/bjo.2008.151365

[8] Hiraoka T, Kakita T, Okamoto F, et al. Long-term effect of overnight orthokeratology on axial length elongation in childhood myopia: a 5-year follow-up study. Invest Ophthalmol Vis Sci.

2012; 53(7): 3913-3919. PMid: 22577080. https://doi.org/ 10.1167/iovs.11-8453

[9] Santodoningo-Rubidp J, Villa-Collar C, Gilmartin B, et al. Myopia control with orthokeratology Contact lemses in Spain: refractive and biometric changes. Invest Ophthalmol Vis Sci. 2012; 53(8): 5060-5065. PMid: 22729437. https://doi.org/10. 1167/iovs. 11-8005

[10] Ophthalmology Group of the Chinese Medical Association. Clinical expert consensus on rigid gas permeable contact lens. Chinese Journal of Ophthalmology. 2012; 48(5): 467-496.

[11] Zhong YY, Zhou XT. Progress in the study of the effect and mechanism of corneal plastic lens in the control of juvenile myopia. Chinese Journal of Ophthalmology and Otorhinolaryngology. 2014; 14(2): 121-123.

[12] Sankaridurg P, Holden B, Smith E, et al. Decrease in rate of myopia progression with a contact lens designed to reduce relative peripheral hyperopia: one-year results. Invest Ophthalmol Vis Sci. 2011; 52(13): 9362-9367. PMid: 22039230. https://doi.org/ 10.1167/iovs.11-7260

[13] Stone RA, Flitcroft DI. Ocular shape and myopia. Ann Acad Med Singapore. 2004; 33(1): 7-15. PMid: 15008555.

[14] Queirós A, González-Méijome JM, Jorge J, et al. Peripheral refraction in myopic patients after orthokeratology. Optom Vis Sci. 2010; 87(5): 323-329. https://doi.org/10.1097/OPX. Ob013e3181d951f 7

[15] Niu Y, Li B, Ji N. Clinical observation of corneal plastic lens in the treatment of moderate myopia in adolescents. Chinese Journal of Optometry Ophthalmology and Visual Science. 2014; 16(2): 92-94.

[16] Huang J, Qu XM, Chen Zhi, et al. The difference between RGPCL, Ortho-K and frame glasses in adolescent myopia was observed after 
one year. Chinese Journal of Optometry Ophthalmology and Visual Science. 2010; 12(1): 33-36.

[17] $\mathrm{Wu} \mathrm{XY,} \mathrm{Liu} \mathrm{SZ.} \mathrm{The} \mathrm{changes} \mathrm{of} \mathrm{the} \mathrm{regulatory} \mathrm{set} \mathrm{and} \mathrm{the} \mathrm{regulation}$ ratio after laser in situ keratomileusis in myopia. Chinese Journal of
Ophthalmology. 2003; 39(3): 132-135. PMid: 12880567.

[18] Xie PY, Wang ZX, Chi H. Observation of long-term corneal plastic effect and safety of myopia in children. Chinese Journal of Strabismus \& Pediatric Ophthalmology. 2008; 16(4): 145-152. 